\title{
Effect of fabrication technique on the crystalline phase and electrical properties of PVDF films
}

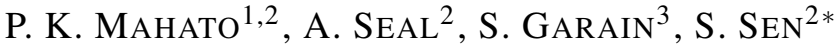 \\ ${ }^{1}$ Academy of Scientific and Innovative Research, CSIR-CGCRI, Kolkata 700032, India \\ ${ }^{2}$ Sensor and Actuator Division, Central Glass and Ceramics Research Institute, Kolkata 700032, India \\ ${ }^{3}$ Department of Physics, Jadavpur University, Kolkata 700032, India
}

\begin{abstract}
The effect of different fabrication techniques on the formation of electroactive $\beta$-phase polyvinylidene fluoride (PVDF) has been investigated. Films with varying concentration of PVDF and solvent - dimethyl formamide (DMF) were synthesized by tape casting and solvent casting techniques. The piezoelectric $\beta$-phase as well as non polar $\alpha$-phase were observed for both the tape cast and solvent cast films from X-ray diffraction (XRD) micrographs and Fourier transform infra-red spectroscopy (FT-IR) spectra. A maximum percentage ( $80 \%$ ) of $\beta$-phase was obtained from FT-IR analysis for a solvent cast PVDF film. The surface morphology of the PVDF films was analyzed by FESEM imaging. The dielectric properties as a function of temperature and frequency and the ferroelectric hysteresis loop as a function of voltage were measured. An enhancement in the value of the dielectric constant and polarization was obtained in solvent cast films.
\end{abstract}

Keywords: PVDF; crystallinity; dielectric; remanent polarization

(C) Wroclaw University of Technology.

\section{Introduction}

Nanogenerators (NGs) are an emerging technology that uses piezoelectric materials to efficiently and effectively harvest mechanical energy from an ambient source. Polyvinylidene fluoride (PVDF), a piezoelectric polymer, has been studied extensively for the application as nanogenerator. PVDF is a relatively simple polymer, with the molecular structure $\left(\mathrm{CH}_{2}-\mathrm{CF}_{2}\right)_{n}$. It is a semicrystalline polymer which exists in several forms: alpha (TGTG'), beta (TTTT), and gamma (TTTGTTTG') phases, depending on the chain conformations as trans $(\mathrm{T})$ or gauche $(\mathrm{G})$ linkages. The $\beta$-phase of PVDF is the most broadly studied piezoelectric polymer material [1-3]. Due to its high piezoelectric coefficient, excellent stability and desirable flexibility, PVDF polymer, has been used in a variety of NG designs for mechanical energy harvesting [4-6].

Various efforts have been made to obtain the piezoelectric $\beta$-phase of PVDF. Stretching a PVDF

*E-mail: shrabanee@cgcri.res.in film results in the $\beta$-phase transformation. In this transformation, the polymer chains are realigned in the crystal, so that the trans-planar zigzag (TTT) conformations are induced. This allows the dipoles in the polymer chains to align normal to the direction of the applied stress. The PVDF $\alpha$-phase films can be converted into polar $\beta$-phase by a uniaxial or biaxial drawing operation [7, 8]. By stretching of PVDF films at $90^{\circ} \mathrm{C}$, a maximum content of $74 \%$ of $\beta$-phase was revealed by FT-IR technique [9].

PVDF tapes with predominantly $\beta$-phase, prepared by tape casting of a solution of PVDF and dimethyl formamide (DMF) solution, have been reported [10]. It was demonstrated that high $\beta$-phase content in PVDF films can be obtained from the melt by adding a small quantity of $\mathrm{CoFe}_{2} \mathrm{O}_{4}$ nanoparticles [11]. The nucleation was explained by the electrical interactions due to the presence of negative nanoparticle surfaces that interact with the polymeric $\mathrm{CH}_{2}$ groups having a positive charge density. This interaction induced the polymer chains to align on the surface of the nanoparticles in an extended TTTT conformation, resulting in formation of the 
$\beta$-phase PVDF. Dense $\beta$-phase dominant PVDF thin films have been obtained by spin coating of PVDF/DMF solution with addition of $\mathrm{Mg}$ $\left(\mathrm{NO}_{3}\right)_{2} \cdot 6 \mathrm{H}_{2} \mathrm{O}$ and drying at $100{ }^{\circ} \mathrm{C}$. A crystallization mechanism was proposed, in which the $\mathrm{Mg}$ $\left(\mathrm{NO}_{3}\right)_{2} \cdot 6 \mathrm{H}_{2} \mathrm{O}$ functioned as the nucleation sites for PVDF crystallization. The intermolecular hydrogen bonding between $\mathrm{Mg}\left(\mathrm{NO}_{3}\right)_{2} \cdot 6 \mathrm{H}_{2} \mathrm{O}$ and PVDF, possibly together with dipolar interaction between PVDF and DMF, promoted the $\beta$-phase formation [12]. Further, the addition of nanofillers is often performed aiming at the nucleation of the electroactive $\beta$-phase of the polymer. The dispersion of nanometer clay layers in PVDF induces heterophase nucleation during crystallization at a higher temperature, which results in the $\beta$-phase formation in PVDF/clay nanocomposites, whose form is stable even after annealing [13].

In this paper, the authors aimed to investigate the effect of different synthesis techniques on the fraction of $\beta$-phase, degree of crystallinity and surface morphology of the PVDF films. Further, the dielectric properties and ferroelectric hysteresis loop were analyzed to correlate them with the piezoelectric $\beta$-phase formation. This gives a basic understanding of the formation of $\beta$-phase in PVDF films by different synthesis techniques.

\section{Experimental}

PVDF pellets and N,N-Dimethylformamide (Mw $\sim 275000 \mathrm{~g} / \mathrm{mol}$ ) (DMF), obtained from Sigma-Aldrich. PVDF tapes/films, were fabricated by two different techniques, i.e. tape casting and solvent casting. The PVDF pellets were dissolved in $\operatorname{DMF}(10,15$, and $20 \mathrm{w} / \mathrm{v} \%)$ solvent by ballmilling or ultrasonication and/or heating.

Three tapes of different concentrations of PVDF solutions (10, 15, and $20 \mathrm{w} / \mathrm{v} \%) \mathrm{PVDF}$ $(1-3)$ were prepared by using doctor blade with a gap of $0.5 \mathrm{~mm}$ in a Tape Caster (Haiku Tech Inc, USA) at room temperature. The tapes were further dried at room temperature for two days to remove the solvent.

Solvent casting technique was also used to synthesize PVDF films with different concentrations
$(10,15$, and $20 \mathrm{w} / \mathrm{v} \%)(\operatorname{PVDF}(4-6))$. First, PVDF solutions were prepared and then heated at $60{ }^{\circ} \mathrm{C}$ at constant magnetic stirring and finally casted at this temperature for $2 \mathrm{~h}$. The PVDF solutions were finally dried at $120{ }^{\circ} \mathrm{C}$ for 16 hours in an oven to remove the residual solvent. In case of PVDF6 , stretching was performed during casting for enhancing the fraction of $\beta$-phase.

The crystalline phases of all the PVDF samples were obtained by X-ray diffraction at room temperature using an X'pert Pro MPD XRD diffractometer (PANalytical system). The presence of crystalline phases were further confirmed by Fourier transform-infrared spectroscopy (Perkin Elmer) from the absorption bands measured over a range of 400 to $1000 \mathrm{~cm}^{-1}$ at room temperature. The surface morphology of the PVDF samples was obtained by scanning electron microscopy using Supra 35VP (Carl Zeiss) field emission microscope.

The samples were coated with a low temperature curable silver paste for dielectric measurements. The dielectric constant and dielectric loss of the PVDF samples were measured using a HIOKI 3532-50 LCR HiTester in the frequency range of $100 \mathrm{~Hz}$ to $1 \mathrm{MHz}$ at different temperatures from 30 to $120{ }^{\circ} \mathrm{C}$. The ferroelectric hysteresis measurements of the PVDF samples were performed using a hysteresis loop tracer (Radiant Technologies Inc.).

\section{Results and discussion}

PVDF tapes of irregular shapes and sizes, with a varying thickness of 0.3 to $1 \mathrm{~mm}$, were obtained by tape casting. In case of PVDF films prepared by solvent casting, the formed films had regular shapes and sizes with the thickness varying from 0.1 to $0.2 \mathrm{~mm}$.

X-ray diffraction (XRD) patterns were first employed to investigate the change in crystallinity due to the change in synthesis technique. The XRD patterns of the tape cast PVDF films (PVDF $(1-3)$ ) reveal the formation of crystalline phases with both the non-polar $\alpha$ - and polar $\beta$-phases (Fig. 1a). The peaks at $2 \theta=17.7(100), 18.4(020)$ and $19^{\circ}(021)$, respectively, show the presence of $\alpha$-phase and the 


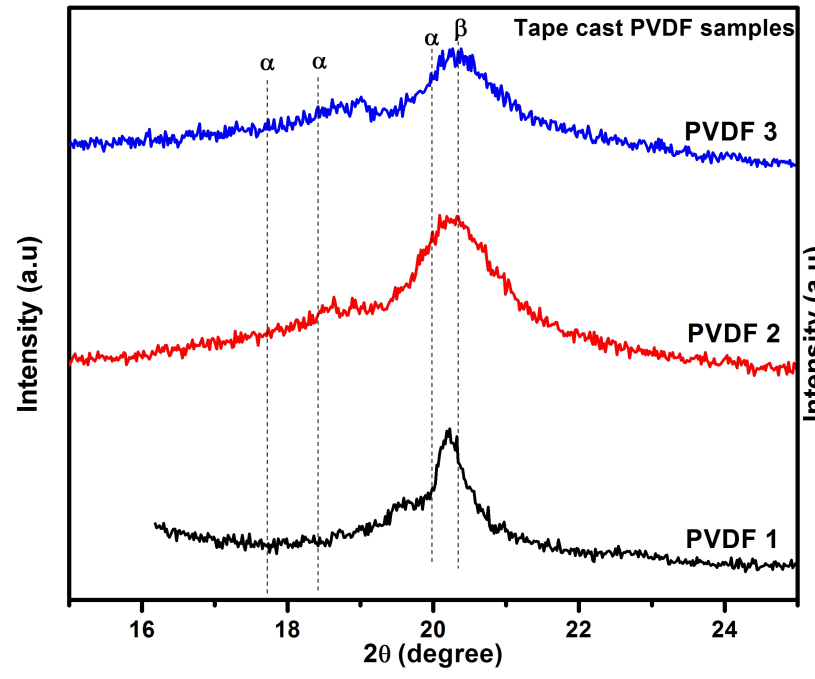

(a)

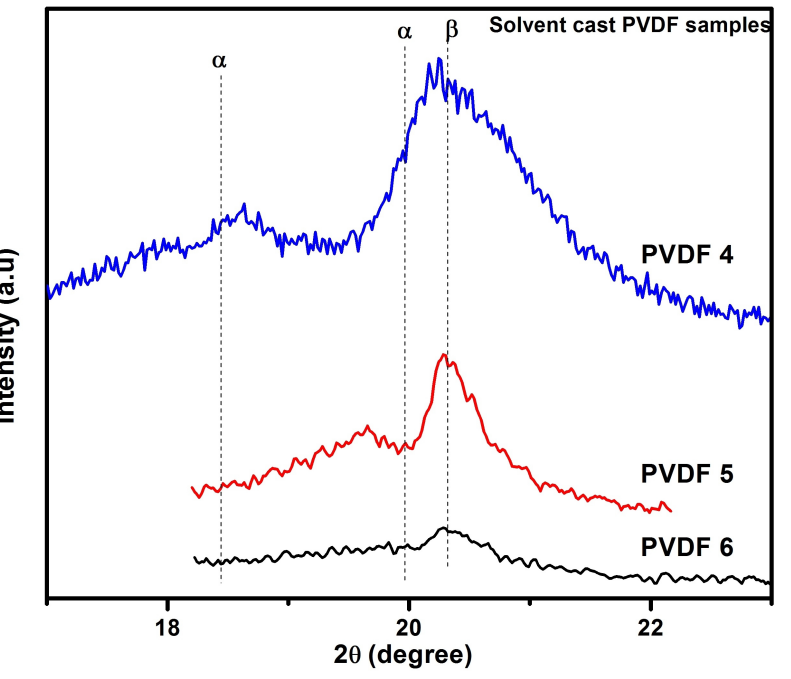

(b)

Fig. 1. (a): XRD patterns of tape cast PVDF films; (b): XRD patterns of solvent cast PVDF films.

peak at $2 \theta=20.3^{\circ}$ refers to the sum of the diffraction in the (110) and (200) planes, which is characteristic of $\beta$-phase [14]. Thus, it was confirmed that both the electroactive $\beta$ - and non-polar $\alpha$-phases of PVDF can be obtained by tape casting.

XRD patterns of the solvent cast PVDF films (PVDF $(4-6)$ ) are shown in Fig. 1b. The most prominent $\beta$ peak $\left(2 \theta=20.3^{\circ}\right)$ is present in all the films along with the presence of $\alpha$ phase ( $2 \theta=$ $18.4^{\circ}$ and $19^{\circ}$ ). The concentration variation, i.e. the ratio of PVDF pellets to DMF has not played any role in the formation of $\beta$-phase. The formation of different phases depends on the preparation technique and the temperature as the existence of the phases $(\alpha, \beta)$ depends on the mobilities of the conformers, which are mainly affected by the thermal energy. If the processing technique and the thermal energy is insufficient to rotate the $\mathrm{CF}_{2}$ groups, there is no trans and gauche conformational change and, hence, formation of $\beta$-phase is not possible [15].

The FT-IR spectra of the PVDF films prepared by tape casting and solvent casting are shown in Fig. $2 \mathrm{a}$ and $2 \mathrm{~b}$, respectively. The absorption bands at 511 and $840 \mathrm{~cm}^{-1}$ are characteristic of the $\beta$-phase, whereas the bands at $408,531,612$, $765,796,855,965 \mathrm{~cm}^{-1}$ are characteristic of the $\alpha$-phase [16].
The presence of both the phases ( $\alpha$ and $\beta$ ) was also observed from FT-IR plots. In order to determine the fraction of the $\beta$-phase present in each sample, IR absorption bands at 766 and $840 \mathrm{~cm}^{-1}$, characteristic of the $\alpha$ - and $\beta$-phases, respectively, the procedure similar to that employed by P. Martin et al. was used [17]. Assuming that the IR absorption follows the Lambert-Beer law, the relative fraction of the $\beta$-phase, $F(\beta)$, (Table 1) can be calculated using the equation 1 :

$$
F(\beta)=\frac{A(\beta)}{1.26 A(\alpha)+A(\beta)}
$$

where the $A(\alpha)$ and $A(\beta)$ are absorbances at 766 and $840 \mathrm{~cm}^{-1}$, respectively.

Table 1. Relative fraction of calculated $\beta$-phase.

\begin{tabular}{cccc}
\hline Sample No. & $\mathrm{A}(\alpha)$ & $\mathrm{A}(\beta)$ & $\mathrm{F}(\beta)(\%)$ \\
\hline \hline PVDF 1 & 3.703 & 3.544 & 43.18 \\
PVDF 4 & 1.356 & 3.4165 & 66.64 \\
PVDF 5 & 0.213 & 0.6695 & 71.36 \\
PVDF 6 & 0.192 & 1.0437 & 81.11 \\
\hline
\end{tabular}

The maximum percentage of $\beta$-phase $(\mathrm{F}(\beta)=$ $80 \%$ ) was obtained for PVDF-6 film, which was due to the effect of stretching. In PVDF/DMF 


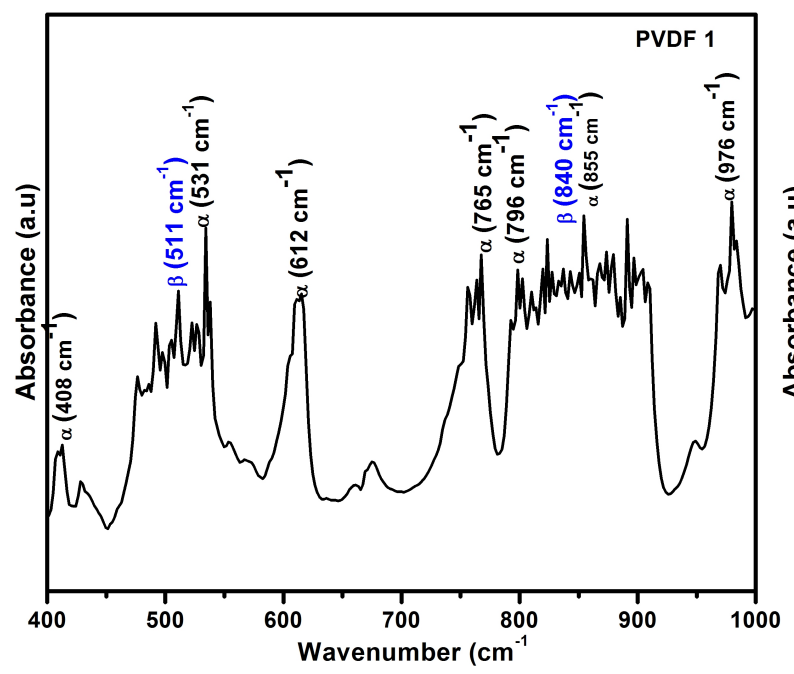

(a)

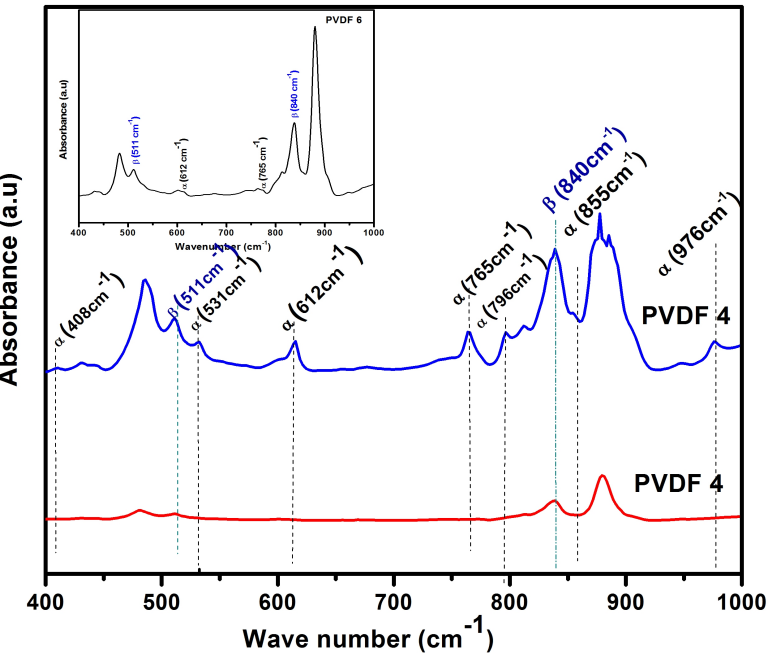

(b)

Fig. 2. (a): FT-IR spectra of tape cast PVDF films; (b): FT-IR spectra of solvent cast PVDF films.

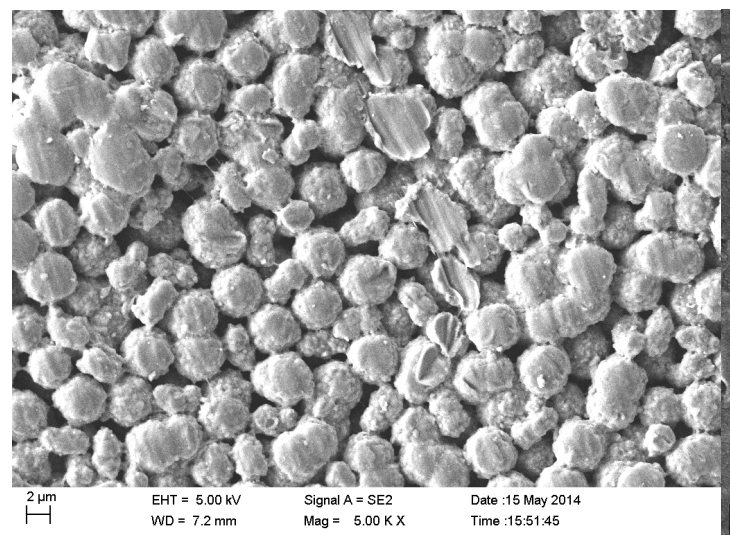

(a)

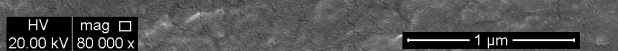

(b)

Fig. 3. (a): FESEM micrographs of tape cast PVDF films; (b): FESEM micrographs of solvent cast PVDF films.

solution, molecular interactions between PVDF and DMF presumably involve dipolar interactions between $\mathrm{C}=\mathrm{O}$ and $\mathrm{CH}_{2}-\mathrm{CF}_{2}$. The presence of weak hydrogen bonding $\mathrm{C}=\mathrm{O} \ldots \mathrm{H}-\mathrm{C}$, disrupts interchain forces of solid PVDF and finally leads to PVDF dissolving [18]. The crystallization of PVDF begins with nucleation, which involves two steps, i.e. the formation of liquid, like clusters of PVDF molecules, and the rate limiting organization of such clusters into crystal nuclei. The dipolar interactions and hydrogen bonding at the interface between PVDF nucleus and DMF molecules can preferably lead to better oriented packing of
$\mathrm{CH}_{2}-\mathrm{CF}_{2}$ dipoles, thus, helping in the formation of trans (TTT) conformation, i.e. $\beta$-phase PVDF. But with relatively large external energy during the drying process, the forces in favor of oriented dipole packing may be too weak to prevent PVDF molecules from being packed into the thermodynamically more stable phase, i.e. $\alpha$-phase. This consideration is consistent with the observation that PVDF can crystallize into either $\beta$-phase or $\alpha$ phase.

The surface morphology of the PVDF films prepared by tape casting (Fig. 3a) and solvent 


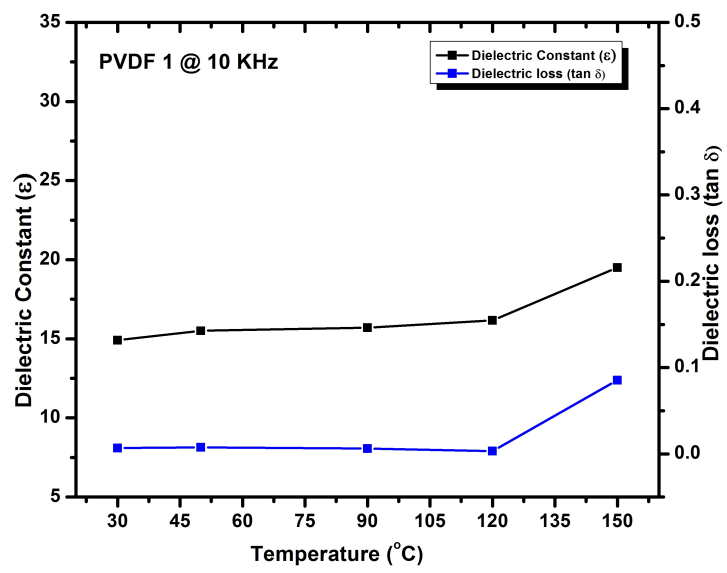

(a)

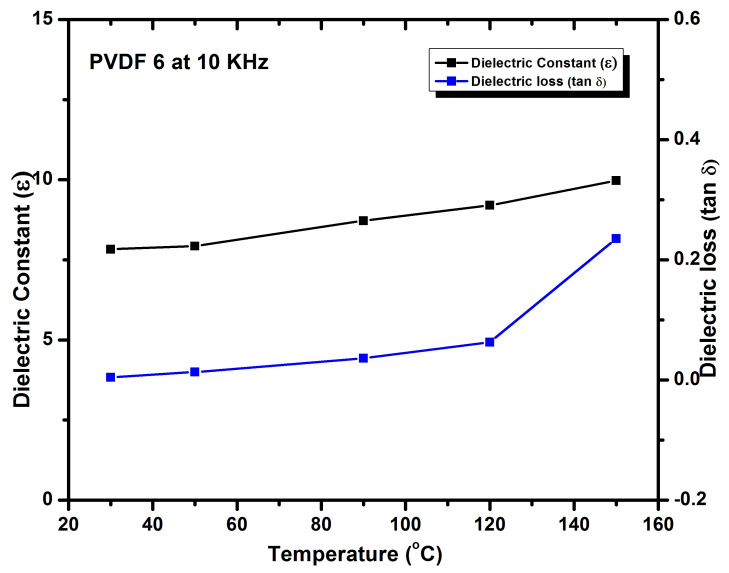

(b)

Fig. 4. (a): Variation of dielectric constant and loss with temperature at $10 \mathrm{kHz}$ for solvent cast PVDF films; (b): Variation of dielectric constant and loss with temperature at $10 \mathrm{kHz}$ for tape cast PVDF films.

casting (Fig. 3b) were analyzed by FESEM imaging of the top surfaces. Both the films, i.e. PVDF $(1-2)$ (Fig. 3a) show similar morphology. Spherulites with the average size of $5 \mu \mathrm{m}$ are observed with pores between two adjacent ones. FESEM micrographs of both PVDF (4 - 5) films (Fig. 3b) shows formation of both spherulites and pores.

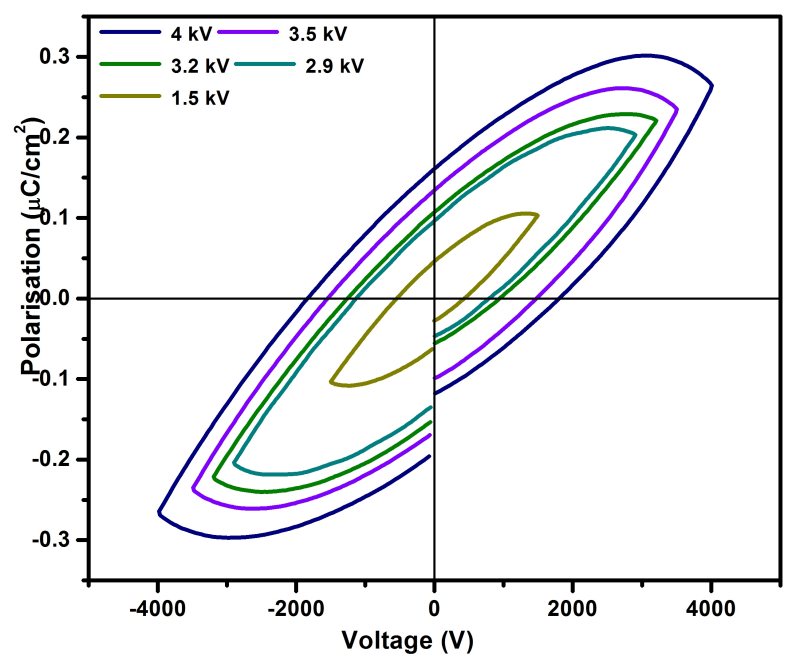

Fig. 5. Hysteresis loop of PVDF-3 film.

The dielectric constant and loss were measured as a function of frequency and temperature for all the PVDF films $(1-6)$. The value of the dielectric constant at room temperature was higher for the film prepared by solvent casting $(\varepsilon=15)$ and it remained more or less constant with frequency. At higher temperatures the value decreased with the increase in frequency. The variation of dielectric constant and loss as a function of temperature at $10 \mathrm{kHz}$ of PVDF films prepared by solvent casting and tape casting are shown in Fig. $4 a$ and $4 b$ respectively. The dielectric properties of a polymer are usually determined by the charge distribution and also by the statistical thermal motion of the polar group [19]. The dipole molecules cannot orient themselves in the lower temperatures but with an increase in temperature the orientation of dipoles is facilitated and this increases the dielectric constant.

Fig. 5 shows the ferroelectric hysteresis loop of a PVDF film for different applied voltages at a frequency of $0.1 \mathrm{~Hz}$. Saturated ferroelectric loop could not be obtained for any of the films as it can only be observed for a poled $\beta$-phase PVDF film. The highest obtained value of remanent polarisation is $0.2858 \mu \mathrm{C} / \mathrm{cm}^{2}$ at an applied voltage of $2 \mathrm{kV}$ at $0.1 \mathrm{~Hz}$ for PVDF-6 film, which may be due to the presence of maximum amount of electroactive $\beta$-phase.

\section{Conclusion}

The effect of preparation technique on crystalline phase and dielectric properties of PVDF 
films fabricated by tape casting and solvent casting was studied. It was observed from FT-IR analysis that a maximum content of $80 \%$ of $\beta$-phase was obtained for the film, which was stretched during solvent casting. The dielectric constant value increased with the increase in the percentage of $\beta$-phase present within the film in case of solvent cast samples. The polarization value of all the films was low as compared with the reported value.

\section{Acknowledgements}

The authors would like to thank Dr A. Sen (Head, Sensor and Actuator Division) and Mr K. Dasgupta (Director, Central Glass and Ceramics Research Institute) for giving permission to publish this paper. Mr P.K. Mahato would like to acknowledge and thank CSIR-CGCRI and Academy of Scientific and Innovative Research (AcSIR) for his fellowship.

\section{References}

[1] Rocha J.G., Gonçalves L.M., Rocha P.F., Silva M.P., MÉNDEZ S.L., IEEE T. Ind. Electron., 57 (2010), 813.

[2] Lei Y., Peggy C., Polymer, 50 (2009), 2133.

[3] Martins P., Caparros C., Gonc,alves R., MarTins P.M., BenelmeKKi M, Botelho G., MendeZ L.S., J. Phys. Chem., 116 (2012), 15790.

[4] Rahman M.A., Lee B.C., Phan D.T., Chung G.S., Smart. Mater. Struct., 22 (2013), 085017.

[5] Chang C., Tran V.H., Wang J., Fuh Y.K., Lin L., Nano Lett., 10 (2010), 726.
[6] Hansen B.J., LiU Y., Yang R., Wang ZL., ACS Nano, 4 (2010), 3647.

[7] Uchino K., Ferroelectric Devices, Marcel Dekker, Inc., New York, 2000.

[8] Vijayakumar R.P., Khakhar D.V., Misra A., J. Appl. Polym. Sci., 117 (2010), 3491.

[9] Salimi A., Yousefi A.A., Poly. Test., 22 (2003), 699.

[10] Seema A., Dayas K.R., Varghese J.M., J. Appl. Polym. Sci., 106 (2007), 146.

[11] Martins P., Costa C.M., Ferreira J.C.C., Mendez S.L., J. Phys. Chem. B, 116 (2012), 794.

[12] He X., Yao K., App. Phys. Lett., 89 (2006), 112909.

[13] Priya L., Jog J.P., J. Appl. Polym. Sci., 89 (2003), 2036.

[14] Gregorio R.J., Ueno E.M., J. Mat. Sci., 34 (1999), 4489.

[15] Gregorio R.J., Cestari M., J. Appl. Polym. Sci. B: Poly. Phy., 32 (1994), 859.

[16] Kobayashi M., Tashiro K., Tadokoro H., Macromol., 8 (1975), 158.

[17] Benz M., Euler W.B., Gregory O.J., Langmuir, 17 (2001), 239.

[18] Yadav V.S., Sahu D.K., Singh Y., DhubKarya D.C., Proceedings of International Conference of Engineers and Computer Scientist, 2010.

[19] Satapathy S., Pawar S., Gupta P.K., Varma K.B.R., Bull. Mater. Sci., 34 (2011), 727. 